\title{
EVALUACIÓN DE PARÁMETROS OCULARES MEDIANTE ULTRASONOGRAFÍA EN CABALLOS CRIOLLOS COLOMBIANOS
}

\author{
Evaluation of ocular parameters by ultrasonography in Colombian
}

\author{
Renso Sneider Gallego Rodríguez ${ }^{1}$ \\ E-mail: renso1287@gmail.com*
}

\author{
Criollo horses. \\ (D) Jesika Leysner Tavera ${ }^{2^{*}}$ \\ E-mail: cabenavides@udenar.edu.co \\ Juan Diego Zuleta Villa ${ }^{3}$ \\ E-mail: juan.zuletav@uam.edu.com
}

${ }^{1}$ MVZ, Esp, Ms.c, Grupo de Investigación Veterinaria GISCA, Facultad de Medicina Veterinaria y Zootecnia, Institución Universitaria Visión de las Américas, Medellin, Colombia. ${ }^{2}$ Grupo de Investigación Veterinaria GINVER, Facultad de Medicina Veterinaria, Corporación Universitaria Remington, Medellín, Colombia.

${ }^{3}$ Grupo de Investigación Veterinaria GISCA, Facultad de Medicina Veterinaria y Zootecnia, Institución Universitaria Visión de las Américas, Medellín, Colombia.

\section{RESUMEN}

La ecografía ocular en los equinos representa uno de los métodos diagnósticos de uso más común, para la determinación de posibles anormalidades oculares. El abordaje de ultrasonido, mediante el modo B, permite el análisis de las diferentes estructuras anatómicas, de una manera no invasiva; la técnica se indica en pacientes con trauma ocular, opacidad corneal, desprendimiento de retina y sospecha de neoplasias retrobulbares. Para el presente estudio, se tuvo en cuenta un total de seis equinos de la raza criollo colombiano; de sexo indiferente; con una edad establecida entre los 4 a los 12 años y con una condición corporal entre 2,5 y 4 (en una escala de 6). Para cada uno de los animales se realizó una ultrasonografía ocular bilateral, utilizando una técnica transpalpebral, a partir de la cual fueron evaluadas estructuras anatómicas como la córnea, la cámara anterior, el lente, el espacio retrobulbar, la retina y el disco óptico; de estos se hizo una medición específica cuantitativa para cámara anterior, retina y disco óptico. Según los resultados obtenidos, se determinó que la cámara anterior presentó un valor de $4 \mathrm{~mm}( \pm 0,07)$; en la retina se obtuvo un valor de $2,4 \mathrm{~mm}( \pm 0,03)$ y en el disco óptico $4,3 \mathrm{~mm}( \pm$ $0,05)$. Se concluyó que los valores obtenidos para la raza criolla colombiana son similares a los reportados para otras razas.

Palabras clave.

Cámara anterior, criollo colombiano, disco óptico, ecografía ocular, equinos, transpalpebral, retina.

\section{Cómo citar:}

Gallego Rodríguez, R.S., Leysner Tavera, J. y Zuleta Villa, J. D. (2021). Evaluación de parámetros oculares mediante ultrasonografía en caballos criollos colombianos. Revista Facultad de Ciencias Agropecuarias -FAGROPEC. Universidad de la Amazonia. Vol. 13 (2), 146155. https://doi.org/10.47847/fagropec.v13n2a5 


\section{ABSTRACT}

The ocular ultrasound in equines represents one of the most common diagnostic methods used in the determination of possible ocular abnormalities, the ultrasound approach using mode B, allows the analysis of the different anatomical structures in a non-invasive way, the technique It is indicated in patients with ocular trauma, corneal opacity, retinal detachment and suspicion of retrobulbar neoplasms. During the study, a total of six equines of the Colombian Creole breed, of indifferent sex, with an established age between 4 and 12 years old and a body condition between 2.5 and 4 (on a scale of 6) were taken into account. For each of the animals, bilateral ocular ultrasonography was performed using a transpalpebral technique in which anatomical structures such as the cornea, anterior chamber, lens, retrobulbar space, retina, and otic disc were evaluated. From these, specific quantitative measurements were made for the anterior chamber. retina and optic disc. According to the results found, it was determined that the anterior chamber presented a value of $4 \mathrm{~mm}( \pm 0.07)$, in the retina a value of $2.4 \mathrm{~mm}( \pm 0.03)$ was obtained, and in the optical disc $4.3 \mathrm{~mm}( \pm 0.05)$. It was concluded that the values obtained for the Colombian creole breed are similar to those reported in other breeds.

\section{Key words.}

Anterior chamber, colombian creole, optic disc, ocular ultrasound, equine, transpalpebral, retina.

\section{INTRODUCCIÓN}

Las patologías oculares son comunes en el equino (Montes et al., 2016). Estas pueden ser de origen primario, o pueden presentarse de forma secundaria a múltiples patologías sistémicas; de modo común, estas patologías no se evidencian a menos que se realice un adecuado examen ocular específico, por lo que representan un reto para la oftalmología equina (Hughes,2010) (Bauer, 2010). Así mismo, las enfermedades oftálmicas son diversas; dado su origen y su patogenia en los equinos, generalmente se ve comprometida la visión de estos en diferentes grados, dependiendo de las estructuras anatómicas afectadas (Gonzalez, et al.,2001). Por lo anterior, un adecuado abordaje diagnóstico cobra importancia en la interpretación de los hallazgos a nivel ocular (Thangadurai et al., 2010).

Siguiendo esta línea, la evaluación ecográfica en modo B es el método más común de diagnóstico imagenológico en la medicina equina, ya que proporciona una imagen bidimensional en tiempo real, del ojo y de la órbita (Scotty et al., 2004). La ecografía ocular en los equinos es un procedimiento simple y poco invasivo (Marchegiani et al., 2017). A su vez representa una técnica segura y práctica, la cual puede ser realizada con el paciente en estación; en pocos casos se requiere sedación o bloqueo nervioso local (Laus, 2014). La técnica se encuentra indicada en opacidad corneal, blefaritis, la cual impide el examen ocular, también en glaucoma, desprendimiento parcial o completo de la retina y posibles masas retro bulbares (Hallowell et al., 2007).

Debido a la ubicación topográfica del ojo, el procedimiento generalmente se realiza de manera simple, permitiendo un examen sistemático y completo de las estructuras oculares, sin 
desconocer la importancia de una adecuada interpretación de la anatomía ecográfica del ojo (Hughes, 2009). El conocimiento de la evaluación ocular ultrasonográfica permite la determinación de valores normales, útiles, en el examen de estructuras como la cámara anterior, grosor del lente, cámara vítrea, retina y nervio óptico (Meister, et al.,2014). La interpretación de las dimensiones normales del ojo en el equino y sus estructuras internas durante el examen ecográfico, representa una gran utilidad en la evaluación de las alteraciones relacionadas con patologías oftálmicas (Soroori et al., 2009).

Por otra parte, debido a que no hay un estudio de investigación en el cual se recopile la evaluación de las estructuras ecográficas anatómicas oculares, en caballos criollos colombianos, desde el presente estudio se plantea la posibilidad de que este pueda representar un punto de partida, para el diagnóstico ecográfico oftalmológico en esta raza determinada. Por lo tanto, el objetivo de esta investigación es realizar una descripción de los parámetros oculares, mediante ultrasonografía, en caballos criollos colombianos.

\section{MATERIALES Y MÉTODOS}

\section{Animales}

Se tuvo en cuenta caballos criollos colombianos; con edad entre los 4 a los 12 años; con condición corporal entre 2,5-4; los cuales no hayan presentado signos de enfermedad ocular de manera histórica. La evaluación ecográfica fue realizada en seis equinos de raza criollos colombianos, los cuales se encontraban presentes en la Clínica Veterinaria de la Corporación Universitaria Remington.

\section{Criterios de Selección}

Se realizó una evaluación diagnóstica histórica, con el fin de determinar si alguno de los animales objeto de estudió presentó lesiones o signos relacionados con enfermedad ocular; a su vez, se les realizó un examen físico general y otro específico ocular, con el fin de interpretar posibles hallazgos estructurales o funcionales en la visión de los pacientes.

\section{Técnica y evaluación ecográfica}

La técnica ecográfica fue realizada por profesionales expertos y por especialistas en el área de diagnóstico por imagen y en medicina interna de equinos. La técnica ultrasonográfica ocular se hizo con un ecógrafo Emperor medical G30, con una sonda lineal a una frecuencia de $12 \mathrm{MHz}$. La evaluación ecográfica ocular se realizó a través de un examen sistemático de todas las estructuras anatómicas, teniendo en cuenta la córnea, la cámara anterior, el cristalino, el iris y los cuerpos ciliares, el humor vítreo, la retina, el disco óptico y el espacio retrobulbar.

Los equinos fueron examinados mediante un abordaje transpalpebral. No fue necesaria la sedación, debido al temperamento dócil de los animales, ya que estos estaban acondicionados al manejo con el personal de la clínica. Se utilizaron exploraciones longitudinales y transversales, con el fin de evaluar las diferentes estructuras anatómicas oculares. Al finalizar 
el examen se lavó el ojo con solución salina fisiológica estéril y, seguido de esto, se realizó una limpieza con toallas de papel.

\section{Artefactos ecográficos}

Durante la evaluación ecográfica ocular la presencia de artefactos es común; las reverberaciones son frecuentes, debido a la interposición del aire entre la sonda lineal y la superficie cóncava del ojo; esta se visualiza como interlineas ecogénicas paralelas, que adquieren cierta profundidad en la imagen ecográfica. El refuerzo acústico se hace visible mediante una porción hiperecogénica, ubicada bajo una estructura anecogénica, o con alta proporción de fluido. Debido a que no hay ninguna atenuación en las ondas ultrasonográficas se produce una señal con mayor intensidad, en la interfase siguiente.

\section{Medición parámetros oculares}

Todas las mediciones fueron realizadas por la misma persona, con el fin de evitar sesgos en los valores obtenidos. Para el estudio se tuvo en cuenta la medición de parámetros relacionados a la cama anterior, la retina y el disco óptico. Se midió la profundidad de la cámara anterior, teniendo en cuenta la porción intermedia del aspecto dorsal entre ésta y el lente de la cápsula anterior. La retina se evaluó en fondo de ojo, realizando una medición simple de su grosor; en el disco óptico se tomó una medida en eje longitudinal y en eje transverso; promediando los dos valores se obtuvo el diámetro de este.

\section{Análisis estadístico}

Se realizaron medidas de estadística descriptiva, a partir de las cuales se efectuó la evaluación de los parámetros oculares, por medio de una base de datos categorizada en hoja de cálculo Excel.

\section{RESULTADOS Y DISCUSIÓN}

En la evaluación ultrasonográfica de los pacientes se encuentran características ecográficas, relacionadas con las estructuras anatómicas evaluables (Tabla 1); la córnea presenta una apariencia delgada de superficie curvilínea e hiperecogénica; la cámara anterior que se encuentra entre la córnea y el lente anterior es de aspecto ovoide anecogénico homogéneo, con

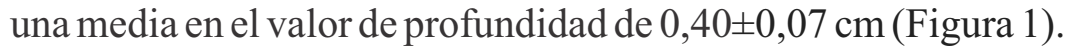

Los cuerpos ciriales se visualizan como estructuras ecogénicas e irregulares, las cuales se encuentran lateralizadas al lente y se extienden desde la periferia hasta el centro del ojo. Dependiendo de la técnica utilizada, es posible la evaluación de la "corpora nigra", se observa como estructuras ecogénicas, ubicadas sobre la porción anterior del iris (Figura 2).

Los lentes en condiciones normales se evalúan como dos interfases curvilíneas, las cuales se relacionan con el lente de la cápsula anterior y el lente de la cápsula posterior; en su centro se presenta anecogenicidad homogénea. El humor vítreo se encuentra de aspecto anecogénico homogéneo. 
Tabla 1.

Medición de los parámetros oculares relacionados a la cámara anterior, la retina y el disco óptico.

\begin{tabular}{llccc}
\hline Animal & Evaluación Ocular & Cámara Anterior $(\mathbf{c m})$ & Retina $(\mathbf{c m})$ & Disco Óptico (cm) \\
\hline Paciente 1 & Ojo izquierdo & 0,45 & 0,27 & 0,39 \\
& Ojo Derecho & 0,57 & 0,25 & 0,38 \\
Paciente 2 2 & Ojo izquierdo & 0,35 & 0,28 & 0,48 \\
& Ojo Derecho & 0,30 & 0,25 & 0,44 \\
Paciente 3 & Ojo izquierdo & 0,37 & 0,28 & 0,41 \\
& Ojo Derecho & 0,42 & 0,20 & 0,39 \\
Paciente 4 & Ojo izquierdo & 0,40 & 0,20 & 0,41 \\
& Ojo Derecho & 0,35 & 0,26 & 0,49 \\
Paciente 5 & Ojo izquierdo & 0,42 & 0,21 & 0,53 \\
& Ojo Derecho & 0,45 & 0,22 & 0,48 \\
Paciente 6 & Ojo izquierdo & 0,32 & 0,24 & 0,40 \\
& Ojo Derecho & 0,34 & 0,19 & 0,35 \\
\hline Media & & 0,4 & 0,24 & 0,43 \\
D.E. & & 0,07 & 0,03 & 0,05 \\
\hline Nor. & & & \\
\hline
\end{tabular}

Nota. D.E. se refiera a desviación estándar.

Figura 1.

Ecografía ocular transpalpebral.

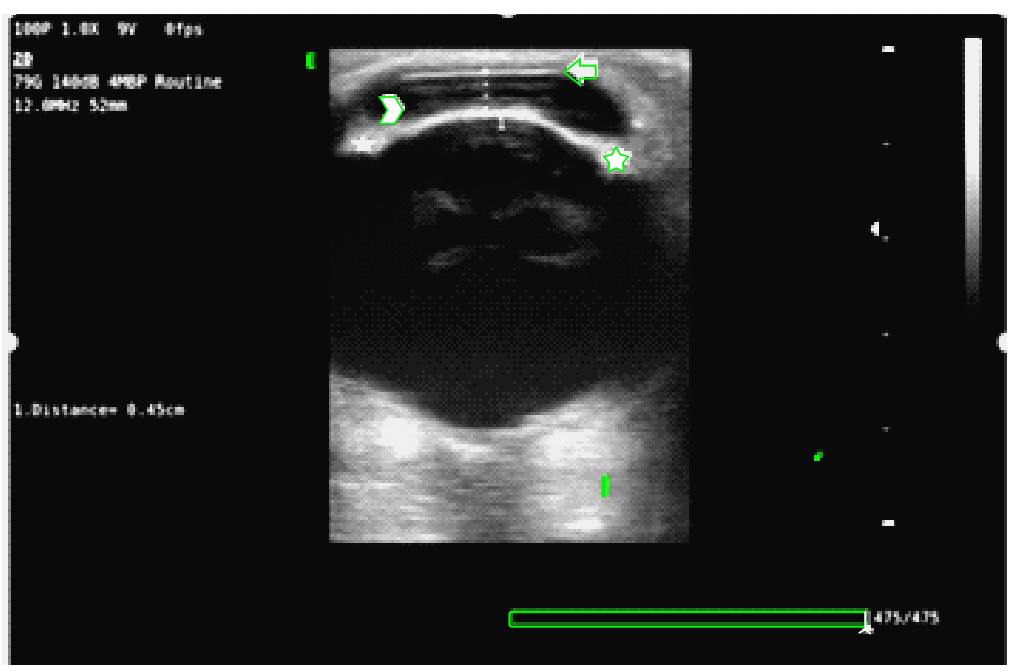

Nota. El abordaje longitudinal deja apreciar estructuras anatómicas normales, en la porción anterior del ojo, córnea (flecha), cámara anterior (punta de flecha), cuerpos ciliares (asterisco). 


\section{Figura 2.}

Ecografía ocular transpalpebral.

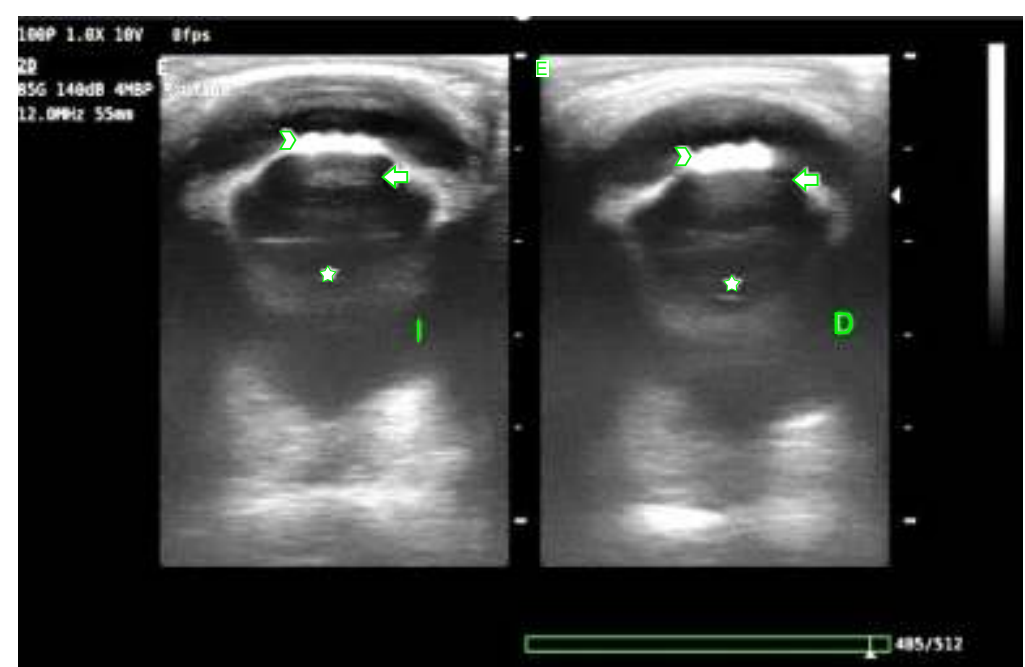

Nota. En abordaje longitudinal se visualizan las estructuras anatómicas intermedias del ojo, se observa corpora nigra (punta de flecha), cápsula anterior (flecha) y cápsula posterior (asterisco).

La retina se identifica como una interfase lineal e hiperecogénica, que recubre el fondo de ojo; en su medición se obtuvo una media en el valor de su grosor de $0,24 \pm 0,03 \mathrm{~cm}$. El nervio óptico se observa en la porción media del fondo de ojo; se encuentra en forma triangular hiperecogénica, en cuyo centro es visible una estructura ovoide hipoecogénica, compatible con el disco óptico, para el cual se obtuvo una media en el valor de su diámetro de $0,43 \pm 0,05$ $\mathrm{cm}^{2}$ (Figura 3).

\section{Figura 3.}

Ecografia ocular transpalpebral.

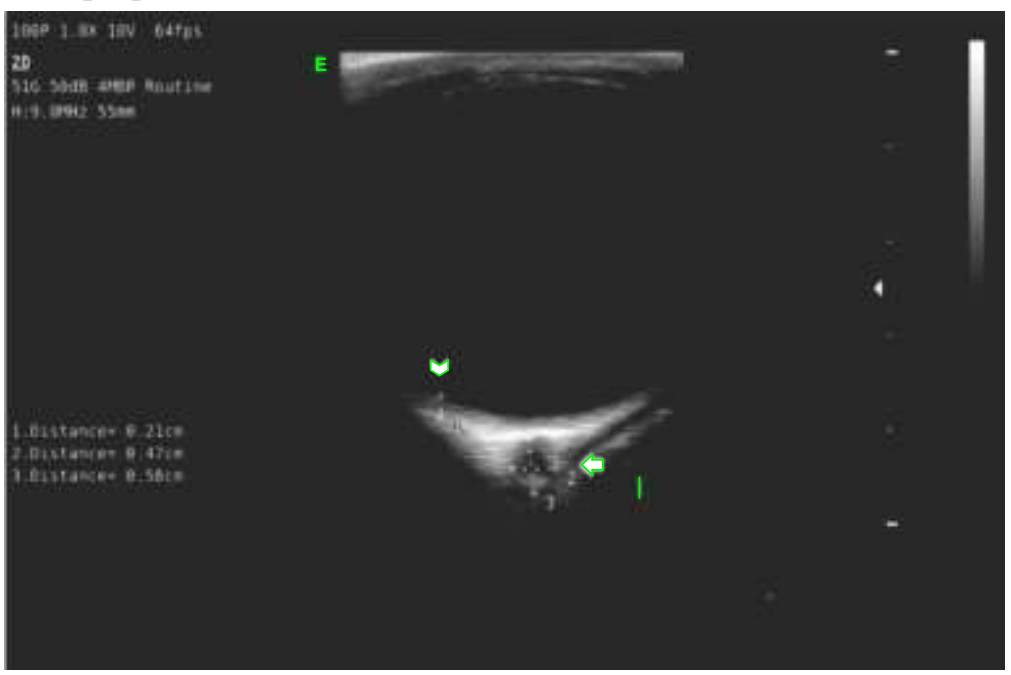

Nota. En abordaje longitudinal se visualizan las estructuras anatómicas posteriores del ojo; es posible observar retina (punta de flecha) $y$ disco óptico (flecha). 
Todos los animales evaluados presentaron un examen clínico ocular normal. El abordaje ecográfico se realizó bajo la técnica transpalpebral, con el fin de proporcionar mayor confort al animal, durante el examen ultrasonográfico; esto asegura que el animal se encuentre tranquilo durante el momento del examen; también garantiza mayor estabilidad en la técnica, así como una visualización adecuada de las estructuras oculares y menor rango en la presentación de artefactos ecográficos. Valentini et al. (2010) mencionan que la técnica transpalpebral genera un menor malestar; a su vez, afirman que una de las desventajas es producida por los mismos párpados, los cuales tienden a facilitar la presentación de artefactos ecográficos, dificultando un adecuado diagnóstico. Finalmente, los autores recomiendan esta técnica en el examen de pacientes con sospecha de lesión corneal (Rogers et al.,1986).

El examen ultrasonográfico ocular hace parte de un conjunto de técnicas, que permiten obtener un diagnóstico confiable (Gonzalez et al., 2001). Autores como Hugues (2009) describen la realización de la ecografía ocular, mediante una frecuencia de entre 7 a $12 \mathrm{MHz}$, mencionando que la variabilidad del rango depende de las estructuras oculares, siendo que se utiliza un menor valor de frecuencia, para evaluar estructuras retrobulbares (Marchegiani et al., 2017). El examen ecográfico ocular, realizado en todos los animales, se hizo bajo una frecuencia de 9 $12 \mathrm{MHz}$, alcanzando una profundidad aproximada entre 52 - $55 \mathrm{~mm}$, obteniendo imágenes con una resolución óptima, inclusive para estructuras retrobulbares, como lo son la evaluación de retina y de disco óptico.

En el presente estudio se realizó la evaluación de las estructuras normales oculares, siendo la córnea uno de los aspectos evaluados. Si bien es cierto que no se realizó una medición de está, sí se evaluó su aspecto ecogénico encontrando que, de forma normal, se visualiza una superficie delgada, curvilínea e hiperecogénica. Soroori et al. (2009) describen la córnea como una interfase hiperecogénica curvilínea relacionada, inmediatamente posterior al párpado. Hallowell (2007) afirma que la mejor manera de observar la córnea es mediante un abordaje transpalpebral y la define como una estructura delgada homogénea y ecogénica (Laus et al.,2014). A su vez, autores como Rogers et al. (1986), afirman que la córnea presenta un espesor de 2,33 $\pm 0,39 \mathrm{~mm}$.

Por su parte, autores como Rogers et al. (1986) afirman que la cámara anterior está compuesta en su interior, por el humor acuoso de aspecto ecográfico anecogénico y homogéneo. Igualmente, describen que la profundidad de la cámara anterior presenta un valor de $3-5 \mathrm{~mm}$ $(4,22 \pm 1,29 \mathrm{~mm})$. Soroori et al. (2009), en un estudio similar, encontraron que el valor medio de la cámara anterior es de $3,0 \mathrm{~mm}$, con una desviación estándar de $0.5 \mathrm{~mm}$. Valores similares fueron encontrados en este estudio, en el cual, respecto de la medición de la cámara anterior, se obtuvo una profundidad media de $4 \mathrm{~mm}( \pm 0,07)$. Gilger (2017) menciona que la evaluación ecográfica anterior del ojo cobra gran importancia, debido a la observación de algunos hallazgos como sinequias, luxación del cristalino, ruptura de la cámara y formación de neoplasias. 
En la evaluación diagnóstico del fondo de ojo pueden encontrarse hallazgos como uveítis posterior, degeneración vítrea, desprendimiento parcial o total de retina y neoplasias retrobulbares (Strobel et al., 2007). La ecografía en modo B es uno de los medios apropiados para las evaluaciones de fondo de ojo (Shlomo, 2017). Gilger (2017) propone un abordaje ecográfico, con una frecuencia cercana a los $7,5 \mathrm{MHz}$, debido a la profundidad de las estructuras anatómicas a evaluar y, a su vez, menciona que esta frecuencia proporciona un detalle adecuado para la imagen. Hallowell y Bowen (1989) describen a la retina en evaluación ecográfica, como una delgada estructura hiperecoica caudal a la cámara posterior, la cual es indiferenciable de la coroides y de la esclera (Thangadurai et al.,2010). Rogers et al. (1986) afirman que el diámetro de la retina se relaciona con valores aproximados de $2 \mathrm{~mm}(2,2 \pm 0,48$ $\mathrm{mm}$ ) (Schiffer et al.,1982). Estos valores se relacionan con los encontrados en el presente estudio, en el cual se estimó que el diámetro de la retina tiene una distancia media de 2,4 $\mathrm{mm}( \pm$ $0,03)$.

Varias estructuras son evaluables en el espacio retrobulbar, incluyendo el nervio óptico, la almohadilla la grasa retrobulbar, los músculos extraoculares y la porción ósea de la órbita (Hallowell y Bowen, 1989). Meister et al. (2014) describen que el nervio óptico puede observarse como una estructura hiperecogénica, la cual se encuentra rodeada de grasa retrobulbar, siendo heterogénea y levemente hipoecogénica. En la presente investigación, al evaluar el disco óptico, se encontró una estructura ovoide hiperecogénica, la cual presentó un diámetro medio de $4,3 \mathrm{~mm}( \pm 0,05)$. La grasa retrobulbar que rodea el disco óptico se evidenció hipoecogénica de ecotextura homogénea. Van den Top et al. (2007) afirman que son varias las indicaciones para realizar la ecografía del espacio retrobulbar; dentro de ellas se pueden mencionar exoftalmo, abscesos, hemorragias y neoplasias (Valentini et al.,2010).

\section{CONCLUSIONES}

Como conclusión del presente estudio, es necesario indicar que este se realiza como la primera aproximación en la ecografía ocular para caballos criollos colombianos. De acuerdo con los resultados encontrados, se recomienda la técnica transpalpebral, con sonda lineal, como ventana de visualización diagnóstica. Los valores y la descripción ecográfica ocular, encontrada en las medidas establecidas para la cámara anterior, para la retina y para el disco óptico, son similares a las reportadas para diferentes razas de la especie. Debido a esto, se considera necesario hacer un estudio, desde el cual se determinen y se estandaricen los valores normales para la raza.

\section{LITERATURACITADA}

Bauer, B.S. (2015). Ocular pathology. Veterinario Clin North Am Equine Pract, 31(2), 425448. https://doi.org/10.1016/j.cveq.2015.04.001

Cunha dos Santos, F. C., Curcio, B., Soares, L., Pazinato, F.M., Soares, P. y Wayne, C. E. 
(2015). Alterações do sistema oftálmico em equinos com ênfase em medidas terapêuticas. A c t a S c i e n t i e Ve t e rin a ri a e, $43,99-106$. https://pesquisa.bvsalud.org/portal/resource/pt/vti-13124

Gilger, B. C. (2017). Advanced Imaging of the Equine Eye. Vet Clin North Am Equine Pract, 33(3),607-626. https://doi.org/10.1016/j.cveq.2017.07.006

Gonzalez, E., Rodríguez, A. y García, I. (2001). Review of ocular ultrasonography. Vet Radiol Ultrasound, 42(6), 485-495.https://doi.org/10.1111/j.1740-8261.2001.tb00975.x

Hallowell, G. D. y Bowen, I. M. (1998). Artifacts and ultrasonographic evaluation of small parts. In Equine Diagnostic Ultrasound. Philadelphia: W.B. Saunders; https://aaep.org/sites/default/files/issues/eve-19-11-Hallowell_eve19-11_lores.pdf

Hallowell, G. D. y Bowen, I. M. (2007). Practical ultrasonography of the equine eye. Equine Veterinary Education., 19(11), 600-605. https://doi.org/10.2746/095777307X254536

Hughes, K. (2009). Ultrasonographic examination of the painful equine eye. In Practice, 31(2), 70-76. https://doi.org/10.1136/inpract.31.2.70

Hughes, K. J. (2010). Ocular manifestations of systemic disease in horses. Equine Veterinary Journal. 42(S37), 89-96. https://doi.org/10.1111/j.2042-3306.2010.tb05640.x

Laus. F., Paggi, E., Marchegiana, A., Cerquetella, M., Spaziante, D., Faillace, V. y Tesei, B. (2014). Ultrasonographic biometry of the eyes of healthy adult donkeys. Veterinary Record, 174(13), 328. http://dx.doi.org/10.1136/vr.101436

Marchegiani, A., Fruganti, A., Cerquetella, M., Cassarina, M. P., Laus, F. y Spaterna, A. (2017). Penetrating palpebral grass awn in a dog. Unusual case of a penetrating grass awn in an eyelid. Journal of Ultrasound, 20(1), 81-84. https://doi.org/10.1007/s40477-016-0234$\underline{1}$

Meister, U., Ohnesorge, B., Kôrner, D. y Boevé, M.H. (2014). Evaluation of ultrasound velocity in enucleated equine aqueous humor, lens and vitreous body. Veterinary Research, 10(1), 250. https://doi.org/10.1186/s12917-014-0250-3

Montes, V. D., Buitrago, J. A. y Cardona, A. J. (2016). Frecuencia de patologías oculares en caballos de vaquería en explotaciones ganaderas del departamento de Córdoba, RECIA, 8 (Supl), 377-385. https://doi.org/10.24188/recia.v8.n0.2016.394

Rogers, M.., Cartee, R. E., Miller, W. y Ibrahim, A. K. (1986). Evaluation of the extirpated equine eye using B-mode ultrasonography. Veterinary Radiology \& Ultrasound, 27(1), 24- 
29. https://doi.org/10.1111/j.1740-8261.1986.tb00616.x

Scotty, N. C, Cutler, T. J, Brooks, D. E., Ferrell, E. (2004). Diagnostic ultrasonography of equine lens and posterior segment. Veteninary Ophthalmology, 7(2), 127-139. https://doi.org/10.1111/j.1463-5224.2004.04009.x

Schiffer, S. P., Rantanen, N. W, Leary, G. A. y Bryan, G. M. (1982). Biometric study of the canine eye, using a-mode ultrasonography. Am J Vet Res, 43(5), 826-830. https://pubmed.ncbi.nlm.nih.gov/7091846/

Shlomo, G. B. (2017). The Equine Fundus. Vet Clin North Am Equine Pract., 33(3):499-517. https://doi.org/10.1016/j.cveq.2017.08.003

Soroori, S., Masoudifar, M., Raoofi, A. y Aghazadeh, M. (2009). Ultrasonographic findings of some ocular structures in Caspian miniature horse. Iranian Journal of Veterinary Research, 10(4). http://ijvr.shirazu.ac.ir/article_1716_c31848eef4439de37ba39d8b03f30eba.pdf

Strobel, B. W., Wilkie, D. A, Gilger, B. C. (2007). Retinal detachment in horses: 40 cases (1998 - 2005). Vet Ophthalmol., 10(6), 380-385. https://doi.org/10.1111/j.1463-5224.2007. $\underline{00574 . x}$

Thangadurai, R., Sharma, S., Bali, D. y Rana, B. P. (2010). Prevalence of ocular disorders in an indian population of horses. Journal of Equine Veterinary Science, 30(6), 326-329. https://doi.org/10.1016/j.jevs.2010.05.001

Van den Top, J., Schaafsma, I., Boswinkel, M. y Klein, W. R. (2007). A retrobulbar abscess as an uncommon cause of exophthalmos in a horse. Equine vet. Educ., 19, 579-583. https://doi.org/10.2746/095777307X254554

Valentini. S., Tamburro, R., Spadari, A., Vilar, J. M. y Spinella, G. (2010) Ultrasonographic Evaluation of Equine Ocular Diseases: A Retrospective Study of 38 Eyes. Journal of Equine Veterinary Science., 30(3). https://doi.org/10.1016/j.jevs.2010.01.058 